\title{
Rheological behavior of polymer nanocomposites filled with spherical nanoparticles: Insights from molecular dynamics simulation
}

\author{
Haoxiang $\mathrm{Li}^{1,2,3}$, Haoyu $\mathrm{Wu}^{1,2,3}$, Wenfeng Zhang ${ }^{1,2,3}$, Xiuying Zhao ${ }^{1,2,3}$, Yangyang Gao ${ }^{1,2,3 *}$, Liqun \\ Zhang $^{1,2,3}$ \\ ${ }^{1}$ Key Laboratory of Beijing City on Preparation and Processing of Novel Polymer \\ Materials, Beijing University of Chemical Technology, 10029, People's Republic of \\ China \\ ${ }^{2}$ State Key Laboratory of Organic-Inorganic Composites, Beijing University of \\ Chemical Technology, 10029, People's Republic of China \\ ${ }^{3}$ Beijing Engineering Research Center of Advanced Elastomers, Beijing University of \\ Chemical Technology, 10029, People's Republic of China
}

\begin{abstract}
It is very urgent to understand the rheological behavior of polymer
\end{abstract} nanocomposites (PNCs) on the molecular level, which is very important for their processing and application. Thus, here the reverse nonequilibrium molecular dynamics simulation is employed to explore it by tuning the nanoparticle (NP) concentration, the polymer-NP interaction and the NP size. The shear viscosity $\left(\eta \sim \dot{\gamma}^{-m}\right)$ exhibits a power law with the shear rate where $\mathrm{m}$ varies from 0.42 to 0.53 at high shear rates. By adopting the Carreau-Yasuda model, the obtained zero-shear viscosity gradually rises with increasing the NP concentration, polymer-NP interaction or reducing the NP size. This is attributed to the strong adsorption of chains by NPs and the formed network, which leads to the retarded dynamics. In addition, both the first and second normal stress differences also show power laws on the shear rates. The chains are gradually extended as the increase of shear rates, which is characterized by the mean-square end-to-end

\footnotetext{
*Corresponding author: gaoyy@mail.buct.edu.cn
} 
distance and the mean square radius of gyration. Especially, the evolution process of the NP network and the polymer-NP network is analyzed to deeply understand the shear thinning behavior. The number of the direct contact structure of NPs increases while the number of polymer-NP bridged structure is reduced. This is further proved by the increase of the formation probability of the NP network and the decrease of the polymer-NP interaction energy. Finally, the chain dynamics is found to be enhanced due to the shear flow. In summary, this work provides a further understanding on the mechanism of the shear thinning of PNCs on the molecular level. 


\section{Introduction}

The incorporation of nanoparticles (NPs) into polymer matrixes can significantly improve their thermal conductivity, electrical conductivity and rheological property. ${ }^{1-3}$ Especially, understanding the rheological property of polymer nanocomposites (PNCs) is very useful for their processing and application. In general, the shear viscosity exhibits a nonlinear relationship with the shear rate, which can describe the rheological behavior of PNCs. It is important to characterize the microstructural change under the shear flow, which can uncover the microscopic mechanism. However, a fundamental understanding is still lacked that deserves our further effect.

To date, lots of works have been contributed to understand the rheological behavior, which is in the service of both polymer processing and advancing our related knowledge. For examples, the graphene oxide (GO) gradually disperses better with increasing the polydimethylsiloxane length, which induces the high critical concentration to form the network. ${ }^{4}$ Meanwhile, the normal stress differences is negative due to the vorticity alignment. In addition, the higher oxygen contents can help the graphene to disperse into the matrix, which exhibits a lower rheological percolation threshold. ${ }^{5}$ After exploring the relationship between the exfoliation degree of clay and the characteristic rheological response, the number of clays particles per unit volume is found to be a key factor for it. ${ }^{6}$ Furthermore, by adopting the Carreau model, both the shear viscosity and power-law behavior rise with increasing the organoclay concentration. ${ }^{7}$ The polymer-silica interaction will affect their packing way and the surrounding chains, which influences the shear viscosity. ${ }^{8}$ In addition, the effect of 
dispersion degree of clay on the rheological properties of PNCs is investigated, which match the nonlinear rheological property well. ${ }^{9,}{ }^{10}$ It is supposed that the rheological behavior of PNCs depends on the network formation and the chain dynamics, which should further understand their molecular origins. As a supplement to experiments, Molecular dynamics (MD) simulation is very powerful in offering the information on the microscopic structure, which help to understand the rheological behavior. By adopting the nonequilibrium MD, the NP-tethering polymers with high interfacial interactions improve both the storage and loss moduli than the homopolymers. ${ }^{11}$ In addition, adding NPs into polystyrene can lead to a non-Einstein-like decrease in viscosity due to to the friction reduction by segment blobs. ${ }^{12}$ The shear viscosity will decrease by orders of magnitude with increasing the shear rate, which is expressed by $\eta \sim \dot{\gamma}^{\mathrm{m}}$. The $\mathrm{m}$ varies about from 0.4 to 0.9 in experiments ${ }^{13}$ while it is from 0.37 to 0.89 in simulations ${ }^{14-17}$. The scaling behavior of viscosity has not reached a consistent conclusion in the shear thinning. Meanwhile, the clear microscopic explanation is lacked for the shear thinning. ${ }^{18,19}$ The flow-induced structure change of chains is considered to be one explanation. ${ }^{14,}{ }^{17,20}$ Furthermore, the dynamics of structural rearrangements in supercooled liquids is analyzed which is closely linked to the rheological behavior. ${ }^{21}$ The results reflect the isotropic rearrangement dynamics in the Newtonian regime. However, it is anisotropic in the non-Newtonian regime. Furthermore, the $\alpha$-relaxation process is progressively suppressed by the shear flow. ${ }^{22}$ Meanwhile, the scaling laws are established for the motion of polymer molecules. Moreover, the frequency of characteristic periodic motion is scaled sublinear with the 
shear rate. ${ }^{23}$

Based on the current progress, a fundamental understanding of the rheological property is still unclear for PNCs. To further understand it, it is very crucial to analyze the change process of both the NP network and the polymer-NP network, which has not been investigated to our knowledge. Here, we employed the reverse nonequilibrium molecular dynamics (RNEMD) simulation to investigate the rheological properties of PNCs subjected to the steady shear flow. Effects of the NP concentration, the polymerNP interaction and the NP size on the shear viscosity, the microscopic structures and the dynamical property are analyzed in details. Their relationship has been established, which can understand the mechanism from the molecular level.

\section{Methods and Models}

In this simulation, a nonequilibrium molecular dynamics simulation is used to explore the rheological behavior of NPs filled PNCs in melted states. The simulated systems consist of linear polymer chains and spherical NPs. Each polymer chain contains 30 beads where each one stands for several monomers. ${ }^{24,25}$ It is noted that the chain length is below the entanglement length aiming to analyze how NPs affect the rheological properties independently. ${ }^{26}$ The mass and diameter are set to be m and $\sigma$ respectively for polymer beads while they are $64 \mathrm{~m}$ and $4 \sigma$ for NP beads. Each system contains 1000 chains. The number of NPs varies from 0 to 480 which is corresponding to their concentration $\varphi$ from $0 \%$ to $29 \%$. In this model, the nonbonded interactions between all beads is described by the expanded Lennard-Jones (LJ) potential, given by 


$$
U_{i j}(r)=\left\{\begin{array}{cc}
4 \varepsilon_{i j}\left[\left(\frac{\sigma}{r-r_{E V}}\right)^{12}-\left(\frac{\sigma}{r-r_{E V}}\right)^{6}\right]+C & r-r_{E V}<r_{\text {cutoff }} \\
0 & r-r_{E V} \geq r_{\text {cutoff }}
\end{array}\right.
$$

where $\mathrm{r}$ means the distance between two beads. The $r_{\text {cutoff }}$ means the cutoff distance which is $2.5 \sigma$ for polymer-polymer interactions and polymer-NP interactions and $1.12 \sigma$ for NP-NP interactions. The polymer-polymer interaction $\varepsilon_{p p}$ and NP-NP $\varepsilon_{\mathrm{nn}}$ are fixed as $1.0 \varepsilon$. The polymer-NP $\varepsilon_{\mathrm{n} p}$ is varied to simulate the different strength. It is noted that the chosen parameters can avoid the aggregation of NPs.

The bonded interaction between the polymer beads is represented by the stiff finite extensible nonlinear elastic (FENE) potential, which is given by

$$
U_{\text {bond }}=-0.5 k R_{0}^{2} \ln \left[1-\left(\frac{r}{R_{0}}\right)^{2}\right]
$$

where $\mathrm{k}$ and $\boldsymbol{R}_{0}$ denote the elastic coefficient and the maximum extensible bond length respectively. They are set as $30 \varepsilon / \sigma^{2}$ and $1.5 \sigma$ to avoid unphysical bond crossing. ${ }^{27,}{ }^{28}$ It is noted that the reduced units are used which simulate a common polymer in our work. The symbols $\mathrm{m}, \sigma$ and $\varepsilon$ mean the units of mass, length, and energy respectively. The time unit $\tau$ is defined as $\tau=\left(m \sigma^{2} / \varepsilon\right)^{1 / 2}$. The reduced units can be corresponding to real units by matching the simulated lateral diffusion coefficient to the experimentally measured value. ${ }^{29,30}$

The shear viscosity $\eta$ is the proportionality constant between a shear rate and a flux of transverse linear momentum, which is defined according to the following constitutive equation

$$
\mathrm{j}_{Y}\left(P_{X}\right)=-\eta \partial v_{X} / \partial Z
$$


It is noted that the shear rate is denoted by the gradient $\partial v_{X} / \partial Y$. The momentum flux $\mathrm{j}_{\mathrm{Y}}$ denotes the $\mathrm{X}$ component of the momentum $\mathrm{PX}_{\mathrm{X}}$, which is transported along the $\mathrm{Y}$ direction in a given time. Currently, the RNEMD technique has been developed by Müller-Plathe. ${ }^{31,32}$ The flux is imposed and the corresponding force is measured during the simulation process. The "reverse" means that the cause and effect are reversed compared to the conventional nonequilibrium simulation methods. This method is proved to be powerful in simulating molecular fluids $s^{32}$, complex fluids ${ }^{33}$, polymers $^{34}$, and ionic liquids ${ }^{35}$. In this RNEMD method, the periodic box is subdivided into $\mathrm{N}$ slabs in the $\mathrm{Y}$ direction. In the 1 st slab moving in the $+\mathrm{X}$ direction, the atom with the largest momentum component in the $-\mathrm{X}$ direction (namely the atom with the smallest $\mathrm{P}_{\mathrm{X}}$ ) is found. Likewise, in the $\mathrm{N} / 2+1$ slab moving in the $-\mathrm{X}$ direction, the atom with the largest momentum component in the $+\mathrm{X}$ direction (namely the atom with the largest $\mathrm{P}_{\mathrm{X}}$ ) is found. Then, the $\mathrm{P}_{\mathrm{X}}$ are exchanged between these two atoms with the same mass. Thus, the total transferred amount of momentum $\mathrm{PX}_{\mathrm{X}}$ can be precisely known. In the steady state, the momentum flux $\mathrm{j}_{\mathrm{Y}}\left(P_{X}\right)$ is obtained:

$$
\mathrm{j}_{Y}\left(p_{X}\right)=P_{X} / 2 t A
$$

where $\mathrm{P}_{\mathrm{X}}$ is the total momentum transferred in a simulation time $\mathrm{t}$ and $\mathrm{A}$ is surface area. In this simulation, the simulation box is divided into 20 bins along the $\mathrm{Y}$ direction. Different exchange frequencies are chosen to get the stable linear velocity profiles. Due to the linear perturbation to the system, a linear velocity profile is obtained and the $\partial v_{X} / \partial Y$ is calculated by a linear regression. The details can be referred to the papers. ${ }^{31,}$ 
Then, a NPs filled PNCs system is constructed with a lower density to generate the initial configuration. The size of the initial simulation box is $120 * 120 * 120 \sigma^{3}$ to avoid the overlapping. Then, the isothermal-isobaric ensemble is adopted to equilibrate the simulation box by adopting the Nose-Hoover temperature thermostat and pressure barostat. Temperature and pressure are chosen to be $T^{*}=1.0$ and $\mathrm{P}^{*}=0.0$ respectively. Velocity-Verlet algorithm is adopted for $5^{*} 10^{7}$ timesteps to realize an equilibrium state with a timestep of $\delta t=0.001 \tau$ for all systems. Periodic boundary conditions are applied during the simulation process. It is noted that the final number density of polymer beads is about 0.85 . To explore the rheological behavior, RNEMD simulations are implemented to calculate the shear viscosity for $30000 \tau$. All of the simulations are carried out by using the large atomic/molecular massively parallel simulator (LAMMPS). ${ }^{36,37}$

\section{Results and discussion}

In this section, we mainly explored the rheological properties and structural changes of NPs filled PNCs by focusing on effects of the NP concentration, the effect of polymer-NPs interactions and the size of NPs.

\subsection{Volume fraction of nanoparticles}

In the application of PNCs, it is necessary to add a large number of NPs into the polymer matrix to reinforce the PNCs, which affects their rheological properties. Thus, in this section, we first focused on the effect of the volume fraction $\varphi$ of NPs on the rheological behavior. First, different exchange frequencies $(v)$ are chosen to obtain proper linear velocity profiles in Fig. S1(a), which obtains the velocity gradient. The 
corresponding visualization of velocity distribution $\left(\mathrm{V}_{\mathrm{X}}\right)$ is shown in Fig. S1(b) for $v=0.1 \tau^{-1}$ as an example. The dependence of the shear viscosity $\eta$ on the shear rate $\dot{\gamma}$ shows a crossover at the critical shear rate $\dot{\gamma}_{\text {c. }}$ It is noted that the shear thinning is observed above $\dot{\gamma}_{\mathrm{c}}$ while a Newtonian plateau appears below it. As shown in Fig. 1,

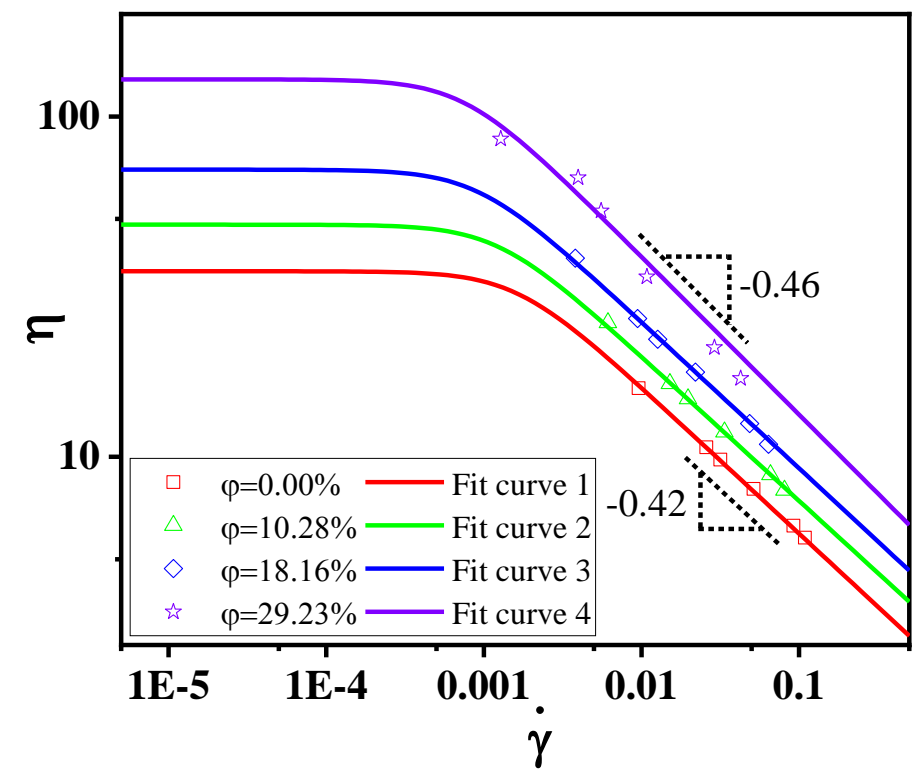

Fig. 1 The shear viscosity $\eta$ with the shear rate $\dot{\gamma}$ for various volume fractions of nanoparticles $\varphi$. The solid lines are the fitting curves by using the Carreau-Yasuda model.

our simulation result reveals that PNCs present a typical shear thinning behavior. The shear viscosity $\eta$ of PNCs gradually decreases with the increase of $\dot{\gamma}\left(\dot{\gamma}>\dot{\gamma}_{\mathrm{c}}\right)$ which is expressed by a universal power law $\eta \sim \dot{\gamma}^{-m}$. The $\mathrm{m}$ varies from 0.42 to 0.46 with the increase of $\varphi$ whose difference is relatively small. It is reported that the relationship between the shear viscosity and the shear rate is $\eta \sim \dot{\gamma}^{-0.44}$ in the simulation work by Baig et $\mathrm{al}^{14}$, which is similar to ours. To obtain the zero-shear viscosity $\eta_{0}$, the individual shear viscosities are fitted by the Carreau-Yasuda model ${ }^{38,39}$ and then obtain $\eta_{0}$ at $\dot{\gamma}=0.0$, which is shown below: 


$$
\eta=\frac{\eta_{0}}{\left[1+(\lambda \gamma)^{2}\right]^{n}}
$$

where $\eta_{0}$ is the zero-shear viscosity, $\lambda$ is a characteristic time constant which is related to the relaxation time of chains, and $\mathrm{n}$ is a power law exponent. We have calculated the $\eta_{0}, \lambda, \mathrm{n}$ and $\dot{\gamma}_{\mathrm{c}}$, for different $\varphi$ which are listed in Table I. It is found

Table I Carreau-Yasuda model parameters and the critical shear rate for different systems in this work

\begin{tabular}{cccccccc}
\hline System & $\varphi$ & $\varepsilon_{\mathrm{n} p}$ & $\mathrm{D}$ & $\mathrm{n}$ & $\lambda$ & $\eta_{0}$ & $\dot{\gamma}_{\mathrm{c}}\left(10^{-4}\right)$ \\
\hline 1 & $0.0 \%$ & 3 & 4 & 0.215 & 621 & 34.4 & 7.6 \\
2 & $10.3 \%$ & 3 & 4 & 0.212 & 820 & 48.2 & 5.2 \\
3 & $18.2 \%$ & 3 & 4 & 0.215 & 1098 & 69.8 & 3.3 \\
4 & $29.2 \%$ & 3 & 4 & 0.232 & 1328 & 128.7 & 2.1 \\
5 & $18.2 \%$ & 2 & 4 & 0.215 & 628 & 39.8 & 10.1 \\
6 & $18.2 \%$ & 5 & 4 & 0.261 & 1501 & 175.6 & 2.5 \\
7 & $18.2 \%$ & 7 & 4 & 0.265 & 3964 & 452.3 & 0.91 \\
8 & $18.2 \%$ & 3 & 1 & 0.263 & 6852 & 656.5 & 0.48 \\
9 & $18.2 \%$ & 3 & 2 & 0.243 & 3518 & 222.8 & 1.2 \\
\hline
\end{tabular}

that the power law exponent $\mathrm{n}$ shows a limited change with increasing the $\varphi$ from 0.215 to 0.232 . The zero-shear rate viscosity $\eta_{0}$ of PNCs exhibits a continuous increase from 34.4 to 128.7 . Meanwhile, the characteristic time constant $\lambda$ gradually rises from 621 to 1328 while the corresponding $\dot{\gamma}_{\mathrm{c}}$ is reduced from $7.6^{*} 10^{-4}$ to $2.1 * 10^{-4}$. Moreover, the $\eta$ of PNCs increases with increasing the $\varphi$ at any shear rate $\dot{\gamma}$ which is larger than that of pure polymers $(\varphi=0.0 \%)$. This is due to the attractive interaction between polymer and NPs, which is consistent with the experimental result. ${ }^{40}$ The shear thinning behavior in PNCs is suggested to be caused mainly by the extension of molecular chains and the destruction of the network structure under the shear flow. It is noted that a lower 
exchange frequency $v$ will lead to the instability of the shear stress. Thus, the Newtonian plateau is not confirmed in the simulation.

Then, we further calculated the first and second normal stress differences which are also important quantities in characterizing the polymers. They are defined as

$$
\mathrm{N}_{1}=-\left(\sigma_{\mathrm{xx}}-\sigma_{\mathrm{yy}}\right)
$$

and

$$
\mathrm{N}_{2}=-\left(\sigma_{\mathrm{yy}}-\sigma_{\mathrm{zz}}\right)
$$

Figure 2 presents the first normal stress difference $\mathrm{N}_{1}$ and the second normal stress differences $\mathrm{N}_{2}$ as a function of the $\dot{\gamma}$ for different $\varphi$. Both $\mathrm{N}_{1}$ and $\mathrm{N}_{2}$ exhibit a
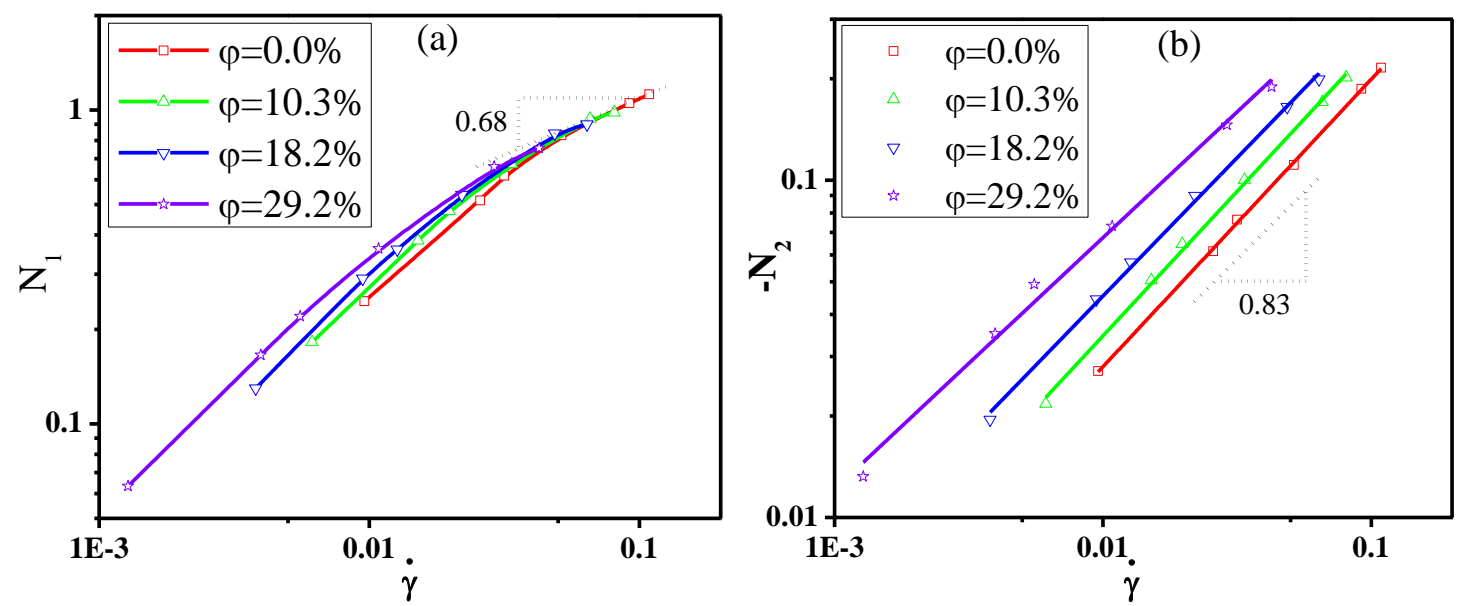

Fig. 2(a) The first normal stress difference $\mathrm{N}_{1}$ and (b) the negative second normal stress difference $\mathbf{N}_{2}$ as a function of the shear rate $\dot{\gamma}$ for various volume fractions of nanoparticles $(\varphi)$.

continuous increase with increasing the $\dot{\gamma}$ and $\varphi$. It is found that the $\mathrm{N}_{1}$ gradually rises with increasing the $\varphi$ at the low $\dot{\gamma}$ while they are approaching the value of the pure polymer at high $\dot{\gamma}$. This can be explained by the change of the chain morphology, the filler network and so on, ${ }^{41}$ which will be also analyzed below. In addition, our simulation reveals that the $\mathrm{N}_{1}$ shows a power law dependence on the $\dot{\gamma}\left(\mathrm{N}_{1} \sim \dot{\gamma}^{0.68}\right.$ at the high $\dot{\gamma}) .{ }^{42}$ From Fig. 2(b), the $\mathrm{N}_{2}$ is much smaller than the $\mathrm{N}_{1}$ for all the systems which 
is difficult to be measured in experiments. Furthermore, the $\mathrm{N}_{2}$ exhibits a power law dependence on $-\mathrm{N}_{2} \sim \dot{\gamma}^{0.83}$ which meanwhile rises with increasing the $\varphi$ at a given shear rate. These exponents' values are consistent with the reported ones. ${ }^{16,42}$

To understand the mechanisms of the shear thinning behavior, the structural change of chains is examined with the $\dot{\gamma}$. The chains' conformation is conveniently measured by calculating the mean-square end-to-end distance $R_{\text {ete }}^{2}$ and the mean square radius of gyration $R_{\mathrm{g}}^{2} \cdot{ }^{16,43}$ Figure 3(a) presents the normalized root-meansquare end-to-end distance $R_{\text {ete }} / L$ with the $\dot{\gamma}$ for different $\varphi$. Here, L is the fully stretched chain length. It is found that the $R_{e t e} / L$ exhibits a continuous increase with increasing the $\dot{\gamma}$. This reflects that the polymer chains are extended along the shear direction. It is reported that the $R_{e t e} / L$ of pure polymer is 0.5 at the $\dot{\gamma} \rightarrow \infty$ from the theories and experiments ${ }^{19,} 23,44-46$, which is higher than our simulated value. Meanwhile, the $R_{e t e} / L$ is enlarged at a higher $\varphi$. This is because the chains can slide and orient more along the shear direction where NPs act as the temporary crosslinking point. ${ }^{47}$ Furthermore, the probability distribution functions (PDFs) of $\mathrm{R}_{\text {ete/ }} \mathrm{L}$, namely $\mathrm{P}\left(\mathrm{R}_{\text {ete }} / \mathrm{L}\right)$, is characterized with the $\dot{\gamma}$ for $\varphi=18.2 \%$ as an example. As shown in Fig. $\mathrm{S} 2$, it is found that the distribution of $\mathrm{P}\left(\mathrm{R}_{\mathrm{ete}} / \mathrm{L}\right)$ is almost the normal distribution for a low $\dot{\gamma}$. As the increase of the $\dot{\gamma}$, the PDF exbibits a decaying peak at small $\mathrm{R}_{\text {ete }} / \mathrm{L}$. Meanwhile, their width becomes larger. This again reflects that polymer chains are stretched with increasing the $\dot{\gamma}$. The $R_{\mathrm{g}}^{2}$ and their three components $R_{\mathrm{gx}}^{2}, R_{\mathrm{gy}}^{2}$ and $R_{\mathrm{gz}}^{2}$ are shown respectively in Fig. 3(b) and Fig. S3 which are normalized by their initial value. It is noted that the value of is nearly equal to 6.0 at equilibrium which is expected 

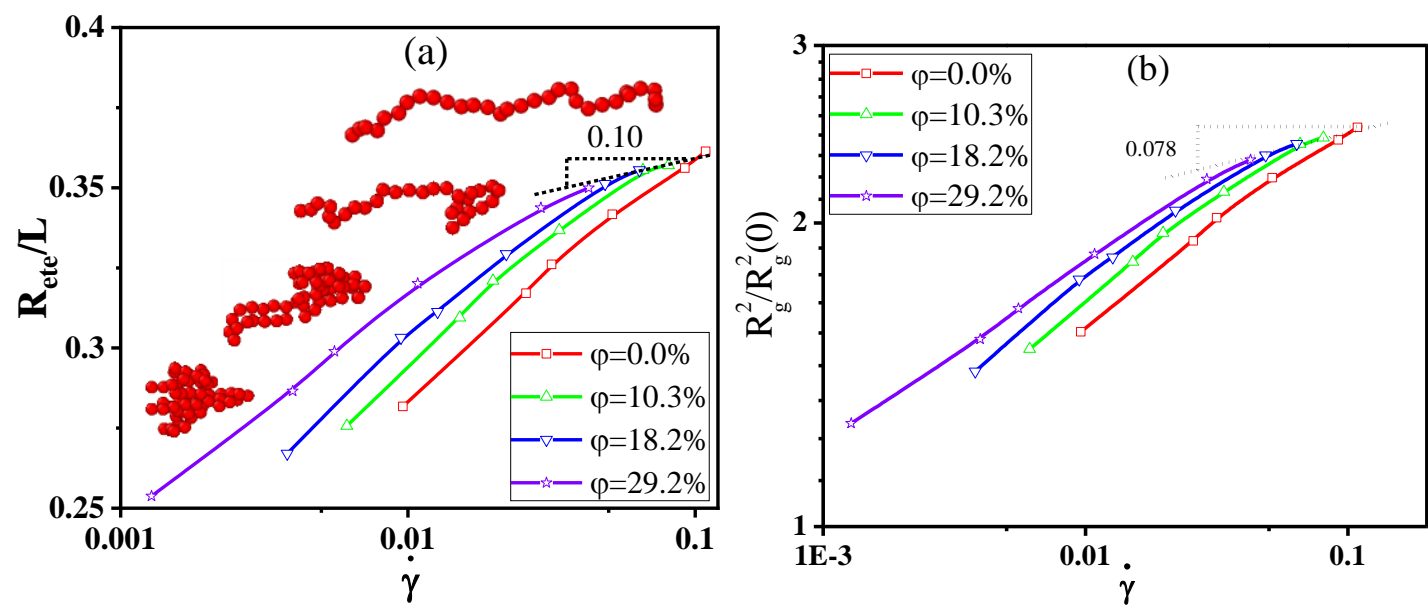

Fig. 3(a) The normalized root-mean-square end-to-end distance $R_{e t e} / L$ and (b) normalized mean square radius of gyration $R_{\mathrm{g}}^{2} / R_{\mathrm{g}}^{2}(0)$ with the shear rate $\dot{\gamma}$ for different volume fraction of nanoparticles $\varphi$.

for Gaussian chains. Meanwhile, the spatial conformation of chains is spherical at $\dot{\gamma}=0.0$ which leads to $R_{\mathrm{gx}}^{2}(0)=R_{\mathrm{gy}}^{2}(0)=R_{\mathrm{gz}}^{2}(0)=R_{\mathrm{g}}^{2}(0) / 3$. As the increase of $\dot{\gamma}$, the chains are stretched along the shear direction and contracted perpendicular to it. Thus, both $R_{\mathrm{g}}^{2}$ and $R_{\mathrm{gx}}^{2}$ rises while the $R_{\mathrm{gy}}^{2}$ and $R_{\mathrm{gz}}^{2}$ decreases quickly. It is noted that their change rate gradually slows down at the high $\dot{\gamma}$ because the number of polymer beads is finite. On the contrary, the dependence of both $R_{\mathrm{g}}^{2}$ and $R_{\mathrm{gx}}^{2}$ on the $\dot{\gamma}$ is described by a power law relationship (namely $R_{\mathrm{g}}^{2} \sim \dot{\gamma}^{0.078}, R_{\mathrm{gx}}^{2} \sim \dot{\gamma}^{0.14}$ at the high $\dot{\gamma}$ ). A similar scaling behavior was also observed for linear polymers. ${ }^{48}$ In addition, both $R_{\text {ete }}$ and $R_{\mathrm{g}}^{2}$ gradually increases with increasing the $\varphi$, which is induced by the attractive interaction between polymer and NPs. Snapshots of some polymer chains are shown in Fig. S4 for different $\dot{\gamma}$, which can prove the results above. Then, the evolution process of the NP network is characterized with the $\dot{\gamma}$ for different $\varphi$. The radial distribution functions (RDFs) of NPs is first calculated to understand how it changes with the $\dot{\gamma}$ in Fig. S5. From RDFs of NPs, it can be found that more NPs aggregate directly to form 
clusters with increasing the $\dot{\gamma}$. To further understand it, the structural characteristic of the NP network is quantified by the maximum cluster size and the cluster number of NPs. Here, if the surface-surface distance of any two NPs is less than $1 \sigma$, they are connected and belong to the same cluster. Meanwhile, $P$ is adopted to reflect the formation probability of the network within the system. Figure 4 presents the change in the maximum cluster size $(\mathrm{S}(\dot{\gamma})-\mathrm{S}(0))$ and the cluster number $(\mathrm{N}(\dot{\gamma})-\mathrm{N}(0))$ as a function of the $\dot{\gamma}$ by their initial values at $\dot{\gamma}=0$.0. It is observed that the $(\mathrm{S}(\dot{\gamma})-\mathrm{S}(0))$ exhibits a continuous increase with increasing the while the $(\mathrm{N}(\dot{\gamma})-\mathrm{N}(0))$ gradually decreases. This indicates that some small clusters are merged into large ones which is consistent with
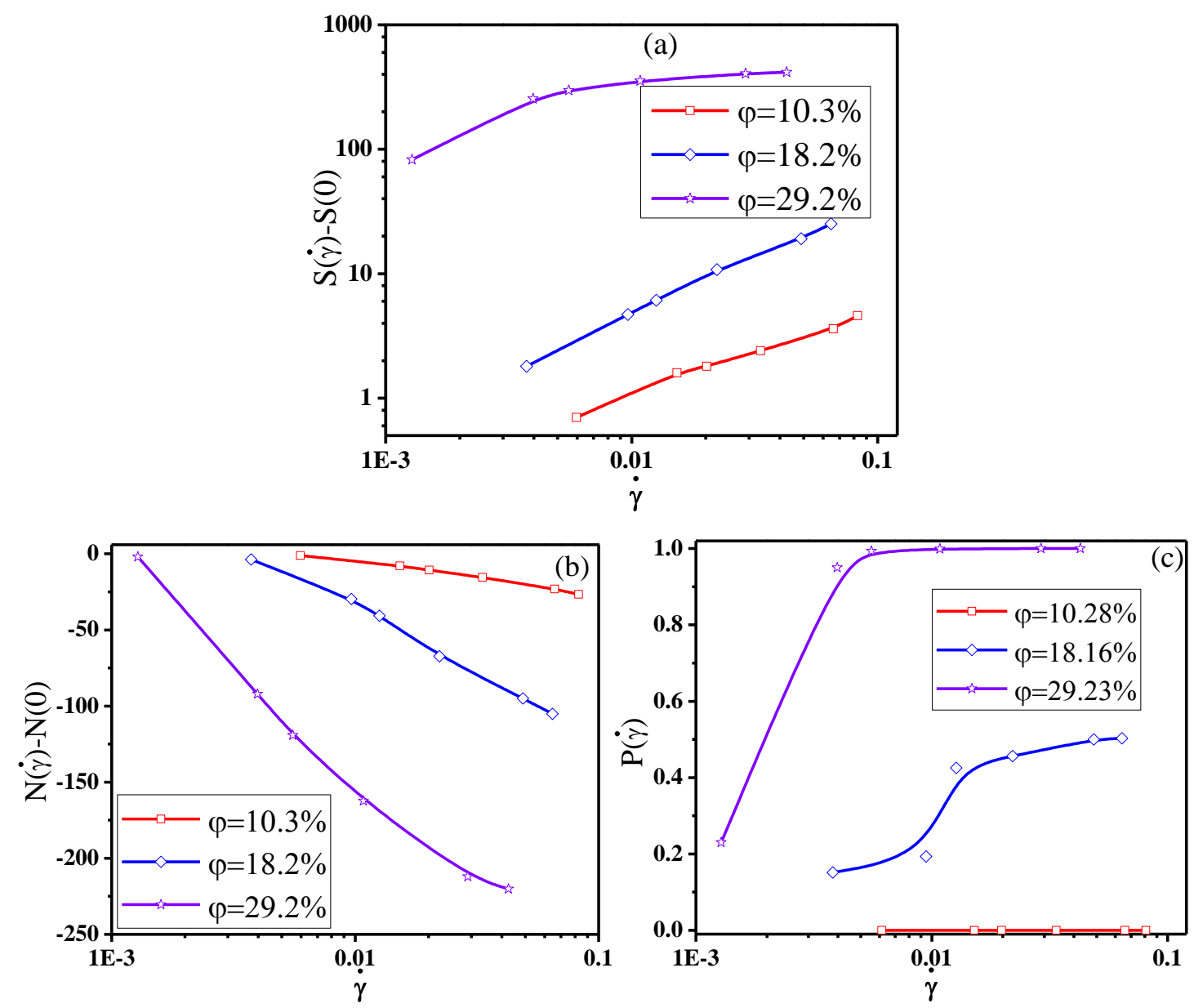

Fig. 4 Change in (a) the maximum cluster size $(\mathrm{S}(\dot{\gamma})-\mathrm{S}(0))$, (b) the cluster number $(\mathrm{N}(\dot{\gamma})-\mathrm{N}(0))$ and (c) the formation probability of the network $P$ with the shear rate $\dot{\gamma}$ for various volume fraction 
of nanoparticles $\varphi$.

previous results that NPs form the direct contact aggregation. As a result, it leads to the increase of the formation probability of the NP network $\mathrm{P}(\dot{\gamma})$ throughout the system. It is noted that another main contribution to the high viscosity is from the polymer-NP network due to the strong interaction. Here, two kinds of structures are used to reflect the change of the polymer-NP network with the $\dot{\gamma}$ in Fig. S5: (i) the direct contact structure of NPs; (ii) the polymer-NP bridged structure via one or two polymer beads. The evolution process of the number of structures (i) and structures (ii) is calculated with the $\dot{\gamma}$. Any pair of NPs belongs to the structure (i) if their distance is between 3.5 $\sigma$ and $5 \sigma$. However, it is the structure (ii) if their distance is between $5 \sigma$ and $6.5 \sigma$. The number of pairs of NPs is used as number of structures. The change in the number of structures (i) $\left(\mathrm{N}_{\mathrm{i}}(\dot{\gamma})-\mathrm{N}_{\mathrm{i}}(0)\right)$ and structures (ii) $\left(\mathrm{N}_{\mathrm{ii}}(\dot{\gamma})-\mathrm{N}_{\mathrm{ii}}(0)\right)$ is presented in Fig. 5. It is found that the $\left(\mathrm{N}_{\mathrm{i}}(\dot{\gamma})-\mathrm{N}_{\mathrm{i}}(0)\right)$ gradually rises
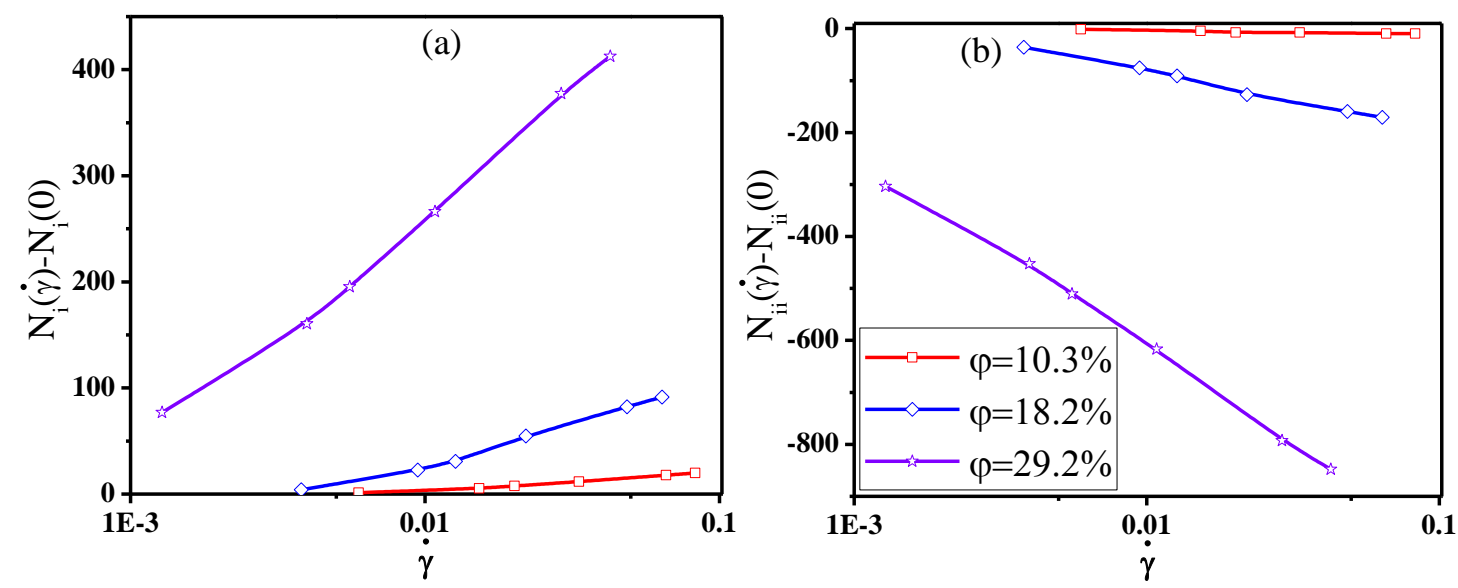

Fig. 5 Change in the number of (a) structures (i) $\left(\mathrm{N}_{\mathrm{i}}(\dot{\gamma})-\mathrm{N}_{\mathrm{i}}(\mathbf{0})\right)$ and $(\mathrm{b})$ structures (ii) $\left(\mathrm{N}_{\mathrm{ii}}(\dot{\gamma})-\mathrm{N}_{\mathrm{ii}}(\mathbf{0})\right)$ with the shear rate $\dot{\gamma}$ for various volume fraction of nanoparticles $\varphi$.

while the $\left(\mathrm{N}_{\mathrm{ii}}(\dot{\gamma})-\mathrm{N}_{\mathrm{ii}}(0)\right)$ decreases with increasing the $\dot{\gamma}$. It indicates that the polymerNP bridged structure via one or two polymer beads is broken down under the flow field. Corresponding to it, the direct contact structure of NPs is formed. Moreover, the change 
in the polymer-NP interaction energy $(\mathrm{E}(\dot{\gamma})-\mathrm{E}(0))$ is characterized to further understand the polymer-NP network in Fig. 6. It is noted that the $\mathrm{E}(0)$ rises with increasing the

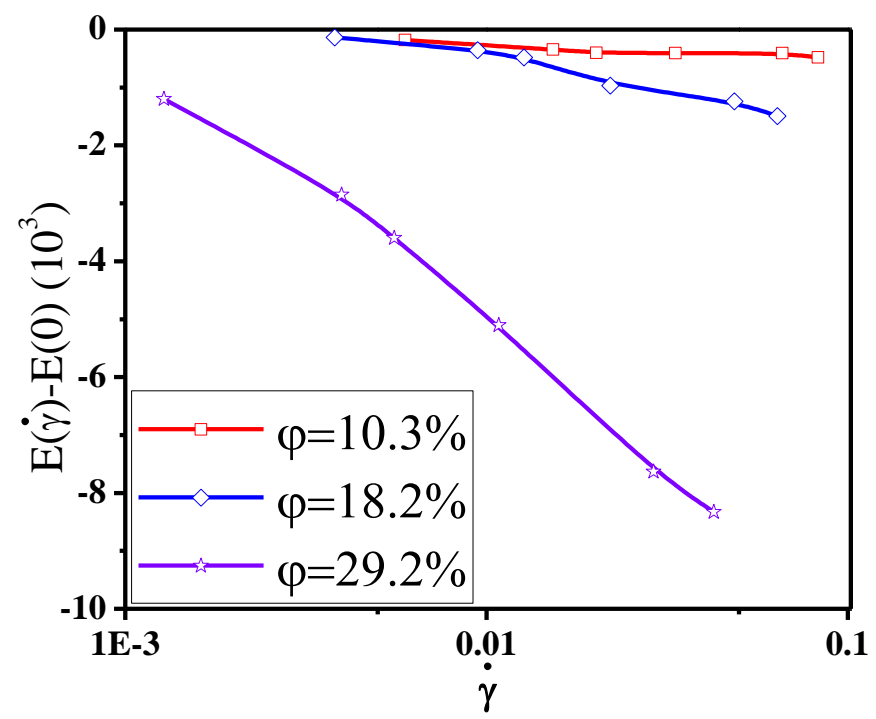

Fig. 6 Change in the polymer-nanoparticles (NP) interaction energy $(\mathrm{E}(\dot{\gamma})-\mathrm{E}(0))$ with the shear rate $\dot{\gamma}$ for various volume fraction of NPs $\varphi$.

$\varphi$, which means the strong polymer-NP network. In addition, the $(\mathrm{E}(\dot{\gamma})-\mathrm{E}(0))$ gradually decreases as the increase of the $\dot{\gamma}$. Meanwhile, the slope is larger at the higher $\varphi$, which reflects a more serious change of networks. Finally, the dynamics of polymer chains is analyzed by calculating the self-part of the intermediate scattering function: $\mathrm{F}_{\text {self }}(\mathrm{q}, \mathrm{t})=\left\langle\frac{1}{\mathrm{~N}} \sum_{\mathrm{j}=1}^{\mathrm{N}} \exp \left\{-\boldsymbol{i} q \cdot\left[\mathrm{r}_{\mathrm{j}}(\mathrm{t})-\mathrm{r}_{0}(0)\right]\right\}\right\rangle \cdot{ }^{22}$ Here, $\mathrm{q}$ is the momentum transfer describing the spatial scale; $\mathrm{N}$ is the number of polymer beads; $\left[\mathrm{r}_{\mathrm{j}}(\mathrm{t})-\mathrm{r}_{\mathrm{j}}(0)\right]$ is the displacement of atom $\mathrm{j}$ within the time $\mathrm{t}$. The $\mathrm{q}$ is chosen to be $\mathrm{q}_{0}=6.9$ which reflects the segmental relaxation approximately corresponding to the maximum peak of the static structure factor. Figure S6 presents the intermediate scattering functions $F_{\text {self }}\left(q_{0}, t\right)$ with the simulation time for different $\dot{\gamma}$ at $\varphi=18.2 \%$. It is observed that the decay of $\mathrm{F}_{\text {self }}\left(\mathrm{q}_{0}, \mathrm{t}\right)$ becomes faster with increasing the $\dot{\gamma}$. To quantify it, the relaxation time is obtained by adopting a two-step relaxation function: 
$\mathrm{F}_{\text {self }}\left(\mathrm{q}_{0}, \mathrm{t}\right)=(1-\mathrm{A}) \exp \left[-\left(\mathrm{t} / \tau_{\mathrm{f}}\right)^{\beta_{\mathrm{f}}}\right]+\mathrm{A} \exp \left[-\left(\mathrm{t} / \tau_{\alpha}\right)^{\beta}\right] .{ }^{22} \mathrm{~A}$ and $\tau_{\mathrm{f}}$ are the additional fitting parameters represent a fast $\beta$-relaxation; the $\beta_{\mathrm{f}}$ and $\beta$ are the stretching exponent; $\tau_{\alpha}$ is the $\alpha$-relaxation time. The $\beta$-relaxation process is nearly unaffected by the $\dot{\gamma}$. The dependence of the $\tau_{\alpha}$ on the $\dot{\gamma}$ for different $\varphi$ is given in Fig. 7. It is observed that the $\tau_{\alpha}$ gradually decreases with increasing the $\dot{\gamma}$ which means that the high $\dot{\gamma}$ gradually suppresses $\alpha$-relaxation process. Their relationship can be described by a power law relationship $\tau_{\alpha} \sim \dot{\gamma}^{-0.72}$ at the high $\dot{\gamma}$. Meanwhile, the $\tau_{\alpha}$ rises as the increase of $\varphi$ due to the retarded dynamics of interfacial chains. In total, the chain extension, the networks breakage and the enhanced dynamics of chains are responsible for the variation of the shear viscosity. In addition, the retarded dynamics of chains by NPs enhances the zero-shear viscosity at low $\varphi$. However, the formed networks further strength the zero-shear viscosity at high $\varphi$.

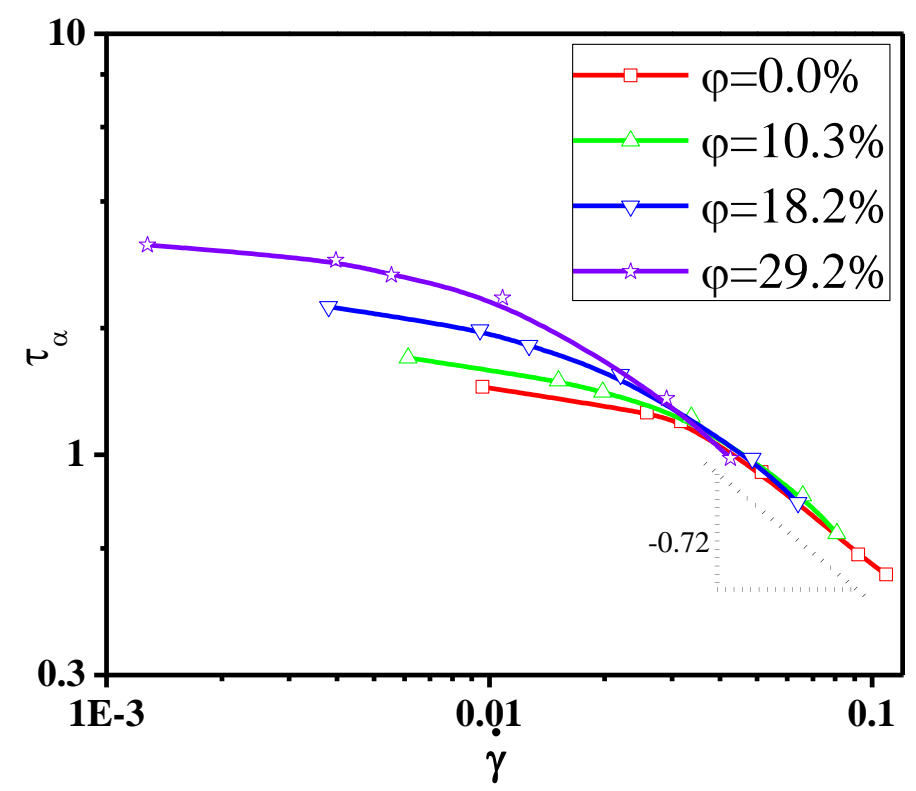

Fig. 7 The relaxation time $\tau_{\alpha}$ as a function of the shear rate $\dot{\gamma}$ for different volume fraction of nanoparticles $\varphi$.

\subsection{Effect of the polymer-nanoparticles interaction}


In experiments, the NPs are chemically modified to improve the compatibility with polymer matrix which is related to the rheological properties of PNCs. Thus, we then turned to understand how the polymer-NP interaction $\varepsilon_{\mathrm{np}}$ affects it by fixing the volume fraction of NPs $\varphi=18.2 \%$. First, the change of the shear viscosity $\eta$ is analyzed with the shear rate $\dot{\gamma}$ which is put in Fig. 8. Similarly, the $\eta$ exhibits a universal power law where the exponent $\mathrm{m}$ varies from 0.42 to 0.53 with increasing the $\varepsilon_{\text {np }}$. Meanwhile, the $\eta$ increases with increasing the $\varepsilon_{\text {np }}$ due to the strong polymerNP network. By adopting the Carreau-Yasuda model, $\eta_{0}, \lambda, \mathrm{n}$ and $\dot{\gamma}_{\mathrm{c}}$ are presented in Table I for different $\varepsilon_{\mathrm{np}}$. It is found that the n shows a limited increase with increasing the $\varepsilon_{\mathrm{np}}$ from 0.215 to 0.265 . The $\eta_{0}$ exhibits a continuous increase from 39.8 to 452.3 . Meanwhile, the $\lambda$ gradually rises from 628 to 3964 while the corresponding $\dot{\gamma}_{\mathrm{c}}$ is

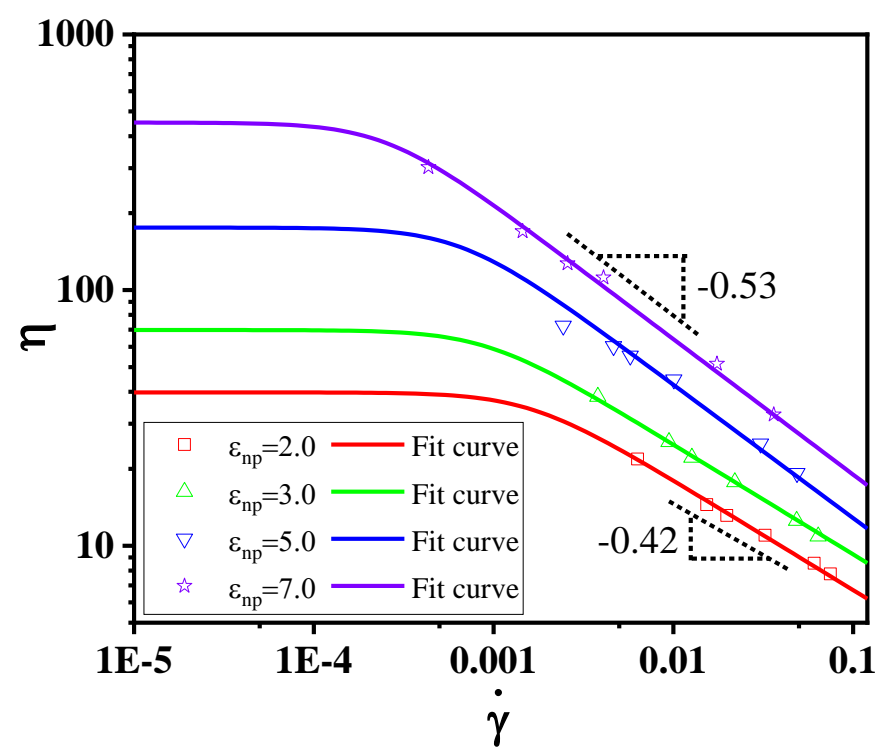

Fig. 8 The shear viscosity $\boldsymbol{\eta}$ with the shear rate $\dot{\gamma}$ for various polymer-nanoparticles (NP) interaction $\varepsilon_{\mathrm{np}}$. The solid lines are the fitting curves by using the Carreau-Yasuda model. reduced from $10.1 * 10^{-4}$ to $0.91 * 10^{-4}$. Then, both the $R_{\text {ete }}^{2}$ and $R_{\mathrm{g}}^{2}$ are calculated which presents a continuous increase with increasing the $\varepsilon_{\text {np }}$ (not shown here). The attractive NPs induces the chain extension along the flow direction which acts as the 
temporary crosslinking points. Importantly, we next characterized the NP network and polymer-NP network to better understand the results. As shown in Fig. S7, it is observed that the change in the maximum cluster size $(S(\dot{\gamma})-S(0))$ gradually rises with increasing the $\dot{\gamma}$ while the $(\mathrm{N}(\dot{\gamma})-\mathrm{N}(0))$ decreases. Meanwhile, the formation probability of the NP network $\mathrm{P}(\dot{\gamma})$ improves. The NPs tend to form the bridged aggregation via polymer chains at the high $\varepsilon_{\text {np }}$, which leads to the increase of $(\mathrm{S}(\dot{\gamma})-\mathrm{S}(0))$ and $\mathrm{P}(\dot{\gamma}) \cdot{ }^{49}$ These results also reflect the change of the NP network. Furthermore, the change in the number of structures (i) $\left(\mathrm{N}_{\mathrm{i}}(\dot{\gamma})-\mathrm{N}_{\mathrm{i}}(0)\right)$ and structures (ii) $\left(\mathrm{N}_{\mathrm{ii}}(\dot{\gamma})-\mathrm{N}_{\mathrm{ii}}(0)\right)$ with the $\dot{\gamma}$ is calculated for different $\varepsilon_{\text {np }}$, which is presented in Fig. 9. The $\left(\mathrm{N}_{\mathrm{i}}(\dot{\gamma})-\mathrm{N}_{\mathrm{i}}(0)\right)$ rises with increasing the $\dot{\gamma}$ while the $\left(\mathrm{N}_{\mathrm{ii}}(\dot{\gamma})-\mathrm{N}_{\mathrm{ii}}(0)\right)$ decreases which reflects the breakage of the
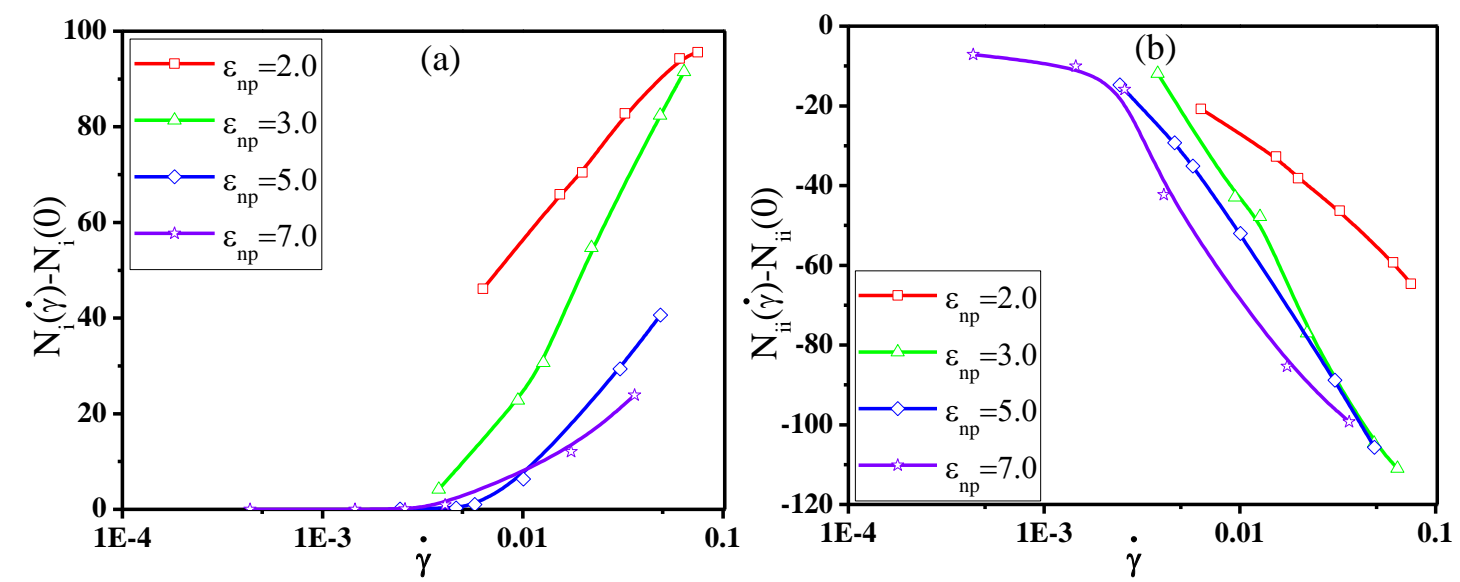

Fig. 9 Change in the number of (a) structures (i) $\left(\mathrm{N}_{\mathrm{i}}(\dot{\gamma})-\mathrm{N}_{\mathrm{i}}(\mathbf{0})\right)$ and $(\mathrm{b})$ structures (ii) $\left(\mathrm{N}_{\mathrm{ii}}(\dot{\gamma})-\mathrm{N}_{\mathrm{ii}}(\mathbf{0})\right)$ with the shear rate $\dot{\gamma}$ for various polymer-nanoparticles (NP) interaction $\varepsilon_{\text {np }}$. polymer-NP network. Meanwhile, the $\left(\mathrm{N}_{\mathrm{i}}(\dot{\gamma})-\mathrm{N}_{\mathrm{i}}(0)\right)$ decreases with increasing the $\varepsilon_{\text {np }}$, while the $\left(\mathrm{N}_{\mathrm{ii}}(\dot{\gamma})-\mathrm{N}_{\mathrm{ii}}(0)\right)$ rises. It is noted that the $\mathrm{N}_{\mathrm{i}}(0)$ is zero at $\dot{\gamma}=0.0$ for $\varepsilon_{\mathrm{np}} \geq 5.0$, which means the inexistence of the structures (i). Similarly, the change in the polymerNP interaction energy $(\mathrm{E}(\dot{\gamma})-\mathrm{E}(0))$ is analyzed. As shown in Fig. S8, the decrease of the $(\mathrm{E}(\dot{\gamma})-\mathrm{E}(0))$ is consistent with the previous results, namely the breakage of the polymer- 
NP network. Meanwhile, the high $\varepsilon_{\text {np }}$ improves the polymer-NP interaction energy, which builds the strong polymer-NP network. Finally, from Fig. S9, the relaxation time $\tau_{\alpha}$ of chains exhibits a decrease with increasing the $\varepsilon_{\mathrm{np}}$ due to their strong adsorption effect by NPs. Generally, the polymer-NP network becomes stronger with higher $\varepsilon_{\text {np }}$, which enhances the zero-shear viscosity. This can be compared to the experimental conclusion that the high interfacial interaction can enhance the shear viscosity due to the adsorbed chains. ${ }^{8,50}$

\subsection{Effect of the nanoparticle size}

Finally, we explored the effect of the NPs size $\left(D_{n p}\right)$ on the rheological properties, which is a common phenomenon in experiments. The polymer-NP interaction and the volume fraction of NPs are set to be 3.0 and $18.2 \%$ respectively. First, the dependence of the shear viscosity $\eta$ on the shear rate $\dot{\gamma}$ is analyzed for different $\mathrm{D}_{\mathrm{np}}$. As shown in Fig. 10, the exponent $\mathrm{m}$ for the universal power law exhibits a decrease from 0.52 to

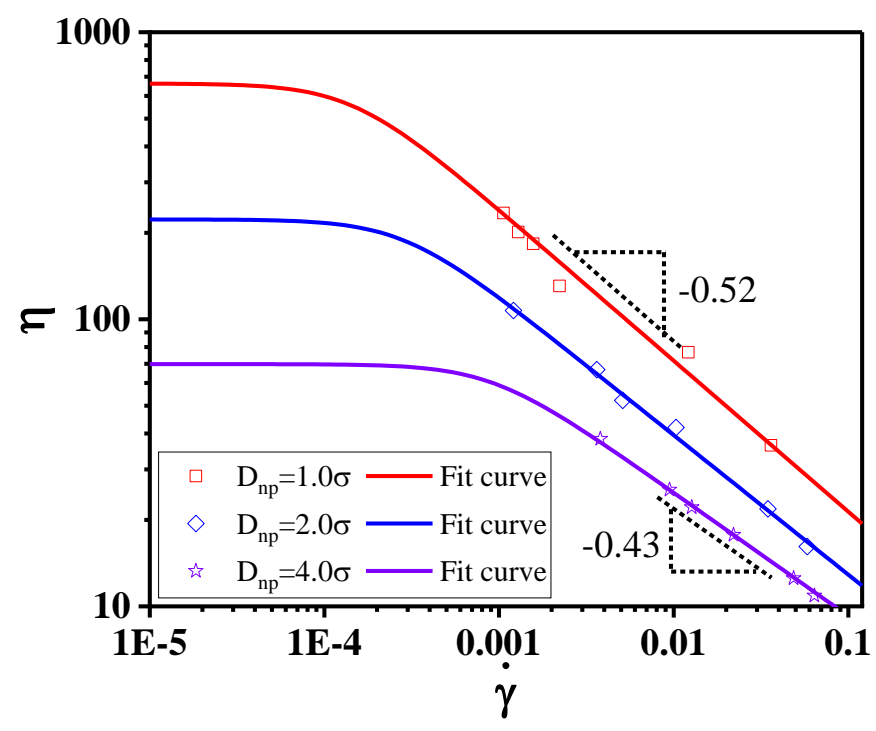

Fig. 10 The shear viscosity $\eta$ with the shear rate $\dot{\gamma}$ for various nanoparticles size $\left(D_{\text {np }}\right)$. The solid lines are the fitting curves by using the Carreau-Yasuda model.

0.43 with increasing the $\mathrm{D}_{\mathrm{np}}$. Meanwhile, the small NPs own a high surface area which 
form a strong polymer-NP network. This leads to the high $\eta$ at the same $\varphi$. According to the Carreau-Yasuda model, the fitted parameters are summarized in Table I for different $\mathrm{D}_{\mathrm{np}}$. As the increase of $\mathrm{D}_{\mathrm{np}}$, the $\mathrm{n}$ declines from 0.263 to 0.215 while the $\eta_{0}$ shows a quick decrease from 656.5 to 69.8 . In addition, it is noted that the $\lambda$ gradually varies from 6852 to 1098 while the corresponding $\dot{\gamma}_{\mathrm{c}}$ changes from $0.48^{*} 10^{-4}$ to $3.3^{*} 10^{-}$ ${ }^{4}$. The $R_{\text {ete }}^{2}$ and $R_{\mathrm{g}}^{2}$ are first analyzed for different $\mathrm{D}_{\mathrm{np}}$ to better understand it. On one hand, the small NPs own the high surface area. On the other hand, it has more NPs at the same $\varphi$ which provide more temporary crosslinking points. As a result, they gradually decrease with improving the $\mathrm{D}_{\mathrm{np}}$ (not shown here). Next, we turned to characterize how the NP network and polymer-NP network change with the $\mathrm{D}_{\mathrm{np}}$ under the flow field. From Fig. S10, the $(S(\dot{\gamma})-S(0))$ shows a gradual increase with increasing the $\dot{\gamma}$ while the $(\mathrm{N}(\dot{\gamma})-\mathrm{N}(0))$ decreases. Meanwhile, the formation probability of the NP network $\mathrm{P}(\dot{\gamma})$ rises. It is noted that the number of NPs becomes small with increasing

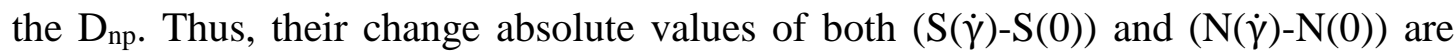
reduced when the $\mathrm{D}_{\mathrm{np}}$ varies from $2.0 \sigma$ to $4.0 \sigma$. However, too many NPs induce their connection with each other which leads to the stability of both $(\mathrm{S}(\dot{\gamma})-\mathrm{S}(0))$ and $(\mathrm{N}(\dot{\gamma})-$ $\mathrm{N}(0)$ ). Similarly, we then analyzed the change in the number of structures (i) $\left(\mathrm{N}_{\mathrm{i}}(\dot{\gamma})-\right.$ $\left.\mathrm{N}_{\mathrm{i}}(0)\right)$ and structures (ii) $\left(\mathrm{N}_{\mathrm{ii}}(\dot{\gamma})-\mathrm{N}_{\mathrm{ii}}(0)\right)$ with the $\dot{\gamma}$ for different $\mathrm{D}_{\text {np. }}$ As shown in Fig. 11 , the $\left(\mathrm{N}_{\mathrm{i}}(\dot{\gamma})-\mathrm{N}_{\mathrm{i}}(0)\right)$ is improved with increasing the $\dot{\gamma}$ while the $\left(\mathrm{N}_{\mathrm{ii}}(\dot{\gamma})-\mathrm{N}_{\mathrm{ii}}(0)\right)$ is reduced. Meanwhile, the change absolute values of both $\left(\mathrm{N}_{\mathrm{i}}(\dot{\gamma})-\mathrm{N}_{\mathrm{i}}(0)\right)$ and $\left(\mathrm{N}_{\mathrm{ii}}(\dot{\gamma})-\mathrm{N}_{\mathrm{ii}}(0)\right)$ 

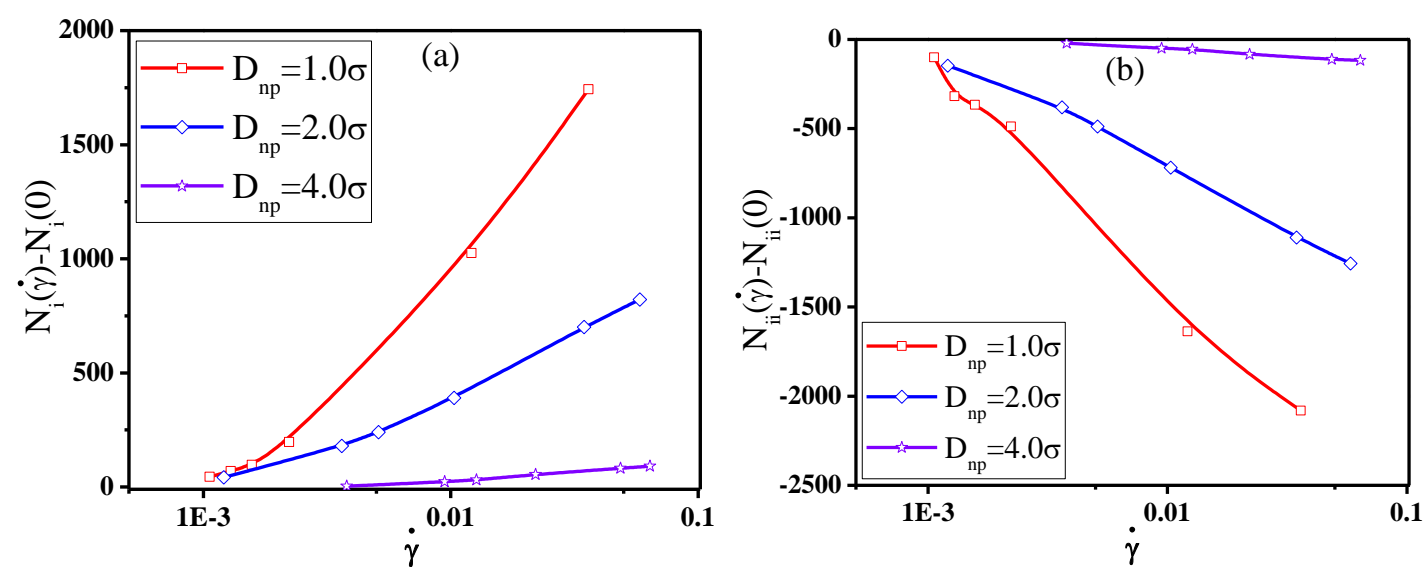

Fig. 11 Change in the number of (a) structures (i) $\left(\mathrm{N}_{\mathrm{i}}(\dot{\gamma})-\mathrm{N}_{\mathrm{i}}(\mathbf{0})\right)$ and $(\mathrm{b})$ structures (ii) $\left(\mathrm{N}_{\mathrm{ii}}(\dot{\gamma})-\mathrm{N}_{\mathrm{ii}}(\mathbf{0})\right)$ with the shear rate $\dot{\gamma}$ for various nanoparticles size $\left(D_{n p}\right)$.

are reduced with increasing the $D_{\text {np }}$. This is due to more NPs for small $D_{n p}$, which induces more significant change of the polymer-NP network. Similarly, the change in the polymer-NP interaction energy $(\mathrm{E}(\dot{\gamma})-\mathrm{E}(0))$ is presented in Fig. S11 which further prove the above results. It is observed that the $(\mathrm{E}(\dot{\gamma})-\mathrm{E}(0))$ is reduced with increasing the $\dot{\gamma}$. Meanwhile, the change absolute values of $(\mathrm{E}(\dot{\gamma})-\mathrm{E}(0))$ is reduced as the increase of the $\mathrm{D}_{\mathrm{np}}$, which reflects the serious breakage of the polymer-NP network. Finally, the relaxation time $\tau_{\alpha}$ of chains gradually declines with increasing the $D_{n p}$ in Fig. S12. These results are consistent with the variation of the shear viscosity.

Currently, the rheological behavior of polymer materials has been explored which is affected by lots of factors, such as the chain structures (the chain length ${ }^{51}$, chain shape $^{52}$ and stiffness of chains ${ }^{53}$ ), the polymer-NP interaction ${ }^{5,8}$, the dispersion degree and concentration of $\mathrm{NPs}^{9,}{ }^{10}$, and so on. The shear viscosity exhibits a power law on the shear rate where the exponent varies from 0.37 to 0.9 at high shear rates. ${ }^{13-17}$ The complex rheological behavior is reported to be related the structure change of polymer chains $^{14,17,20,48}$ and the heterogeneous dynamics under the shear flow. ${ }^{21-23}$ In this work, 
we focused on the rheological property of PNCs by mainly analyzing the evolution process of both the NP network and the polymer-NP network in details, which is the main difference with these works. At last, the relaxation time $\tau_{\alpha}$ of chains is calculated

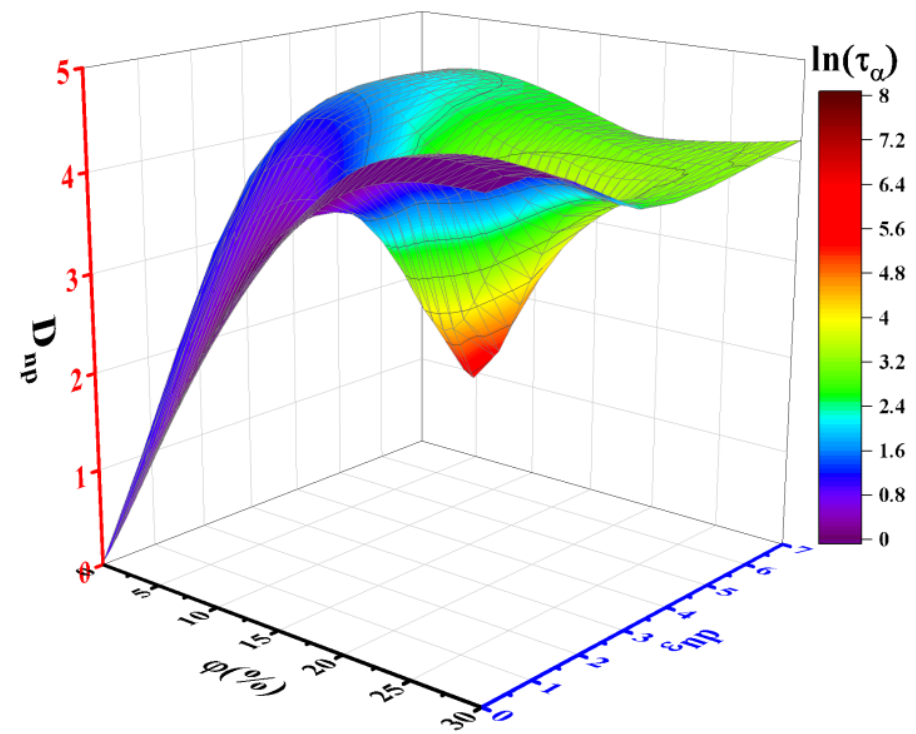

Fig. 12 The relaxation time $\tau_{\mathrm{a}}$ with the volume fraction of nanoparticles (NP) $\varphi$, the polymer-NP interaction $\varepsilon_{\mathrm{np}}$, and the NP size $\left(\mathrm{D}_{\mathrm{np}}\right)$.

at $\dot{\gamma}=0.0$ for different systems in Fig. 12. It is found that the $\tau_{\alpha}$ gradually decreases with increasing the NP concentration, polymer-NP interaction and reducing the NP size, which is consistent with the zero-shear viscosity. This is attributed to the strong adsorption of chains and the formed network, which leads to the retarded dynamics. Especially, the maximum $\tau_{\alpha}$ appears at $\mathrm{D}_{\mathrm{np}}=1.0 \sigma$, which emphasizes the importance of nanosize.

\section{Conclusions}

In this work, the shear viscosity, the structural and dynamical properties of polymer nanocomposites (PNCs) are investigated under the shear flow by RNEMD simulations. The shear viscosity presents an exponential decrease at high shear rates 
where exponent $\mathrm{m}$ varies from 0.42 to 0.53 . The zero-shear viscosity is obtained by the Carreau-Yasuda model, which increases with increasing the NP concentration, polymer-NP interaction and reducing the NP size. Due to the attractive adsorption of chains and the formed network, the dynamics of chains is strong retarded. Furthermore, the dependence of both the first and second normal stress differences on the shear rate can be also described by power laws. The mean-square end-to-end distance and the mean square radius of gyration are used to characterize the structural change of chains, which proves the extended conformation. Importantly, both the NP network and the polymer-NP network are characterized to deeply understand the shear thinning. Our results present that the number of the direct contact structure of NPs gradually rises. However, the number of polymer-NP bridged structure is declined. This is further proved by the increase of the formation probability of the NP network and the decrease of the polymer-NP interaction energy. Finally, the relaxation time of the chain dynamics gradually decreases with the shear rate which is consistent with the shear viscosity. In summary, this work could provide a fundamental understanding on the shear thinning of PNCs on the molecular level.

\section{Acknowledgements}

This work is supported from the National Natural Science Foundation of China (51973012, 21704003), the Foundation for Innovative Research Groups of the NSF of China (51521062). The authors acknowledge the National Supercomputer Center in Guangzhou, Lvliang and Shenzhen. 


\section{References}

1. A. Guimont, E. Beyou, G. Martin, P. Sonntag and P. Cassagnau, Macromolecules, 2011, 44, 38933900 .

2. A. Mora, F. Han and G. Lubineau, Results in Physics, 2018, 10, 81-90.

3. Z. Han and A. Fina, Progress in Polymer Science, 2011, 36, 914-944.

4. R. Niu, J. Gong, D. Xu, T. Tang and Z.-Y. Sun, Polymer, 2014, 55, 5445-5453.

5. H.-B. Zhang, W.-G. Zheng, Q. Yan, Z.-G. Jiang and Z.-Z. Yu, Carbon, 2012, 50, 5117-5125.

6. J. Zhao, A. B. Morgan and J. D. Harris, Polymer, 2005, 46, 8641-8660.

7. Y. H. Hyun, S. T. Lim, H. J. Choi and M. S. Jhon, Macromolecules, 2001, 34, 8084-8093.

8. B. J. Anderson and C. F. Zukoski, Macromolecules, 2008, 41, 9326-9334.

9. M. Kim, H. Y. Song, W. J. Choi and K. Hyun, Macromolecules, 2019, 52, 8604-8616.

10. W. You, W. Yu and C. Zhou, Soft Matter, 2017, 13, 4088-4098.

11. P. Xu, J. Lin and L. Zhang, The Journal of Physical Chemistry C, 2017, 121, 28194-28203.

12. T. Chen, H.-Y. Zhao, R. Shi, W.-F. Lin, X.-M. Jia, H.-J. Qian, Z.-Y. Lu, X.-X. Zhang, Y.-K. Li and Z.-Y. Sun, Nature Communications, 2019, 10, 5552.

13. R. B. Bird, R. C. Armstrong and O. Hassager, Dynamics of Polymeric Liquids; Fluid Mechanics, Wiley \& Sons, New York, 1987.

14. C. Baig, V. G. Mavrantzas and M. Kröger, Macromolecules, 2010, 43, 6886-6902.

15. M. Kröger and S. Hess, Physical Review Letters, 2000, 85, 1128-1131.

16. X. Xu, J. Chen and L. An, The Journal of Chemical Physics, 2014, 140, 174902.

17. T. Aoyagi and M. Doi, Computational and Theoretical Polymer Science, 2000, 10, 317-321.

18. A. Subbotin, A. Semenov, E. Manias, G. Hadziioannou and G. ten Brinke, Macromolecules, 1995, 28, 3898-3900.

19. R. G. Winkler, The Journal of Chemical Physics, 2010, 133, 164905.

20. R. Everaers, S. K. Sukumaran, G. S. Grest, C. Svaneborg, A. Sivasubramanian and K. Kremer, Science, 2004, 303, 823-826.

21. A. Furukawa, K. Kim, S. Saito and H. Tanaka, Physical Review Letters, 2009, 102, 016001.

22. A. Giuntoli, F. Puosi, D. Leporini, F. W. Starr and J. F. Douglas, Science Advances, 2020, 6, eaaz0777.

23. C. M. Schroeder, R. E. Teixeira, E. S. G. Shaqfeh and S. Chu, Physical Review Letters, 2005, 95, 018301.

24. A. Piserchia, M. Zerbetto, M.-V. Salvia, G. Salassa, L. Gabrielli, F. Mancin, F. Rastrelli and D. Frezzato, The Journal of Physical Chemistry C, 2015, 119, 20100-20110.

25. Q. Wang, D. J. Keffer, D. M. Nicholson and J. B. Thomas, Macromolecules, 2010, 43, 10722-10734.

26. R. G. Larson, Journal, 1999.

27. K. Kremer and G. S. Grest, The Journal of Chemical Physics, 1990, 92, 5057-5086.

28. K. Kremer, G. S. Grest and I. Carmesin, Physical Review Letters, 1988, 61, 566-569.

29. M. P. Allen and D. J. Tildesley, Computer Simulation of Liquids, Oxford University: New York, 1987.

30. F. Qu, W. Sun, B. Li, F. Li, Y. Gao, X. Zhao and L. Zhang, Soft Matter, 2020, 16, 10454-10462.

31. F. Müller-Plathe, Physical Review E, 1999, 59, 4894-4898.

32. P. Bordat and F. Müller-Plathe, The Journal of Chemical Physics, 2002, 116, 3362-3369.

33. M. Zhang and F. Müller-Plathe, The Journal of Chemical Physics, 2005, 123, 124502.

34. X. Chen, P. Carbone, W. L. Cavalcanti, G. Milano and F. Müller-Plathe, Macromolecules, 2007, 40, 
8087-8095.

35. W. Zhao, F. Leroy, S. Balasubramanian and F. Müller-Plathe, The Journal of Physical Chemistry B, 2008, 112, 8129-8133.

36. S. Plimpton, Journal of Computational Physics, 1995, 117, 1-19.

37. J. Wang, M. Zhou, D. Lu, W. Fei and J. Wu, Green Energy \& Environment, 2020, 5, 364-373.

38. M. S. Kelkar and E. J. Maginn, The Journal of Physical Chemistry B, 2007, 111, 4867-4876.

39. R. B. Bird, R. C. Armstrong and O. Hassager, Fluid Mechanics I, Wiley, New York, 1987.

40. A. K. Zachariah, V. G. Geethamma, A. K. Chandra, P. K. Mohammed and S. Thomas, RSC Advances, 2014, 4, 58047-58058.

41. R. Krishnamoorti, J. Ren and A. S. Silva, The Journal of Chemical Physics, 2001, 114, 4968-4973.

42. C. M. Schroeder, R. E. Teixeira, E. S. G. Shaqfeh and S. Chu, Macromolecules, 2005, 38, 19671978.

43. Y. Gao, J. Liu, L. Zhang and D. Cao, Macromolecular Theory and Simulations, 2014, 23, 36-48.

44. D. E. Smith, H. P. Babcock and S. Chu, Science, 1999, 283, 1724-1727.

45. H. Tian and P. Tao, J Chem Inf Model, 2020, 60, 4569-4581.

46. H. Tian, F. Trozzi, B. D. Zoltowski and P. Tao, J Phys Chem B, 2020, 124, 8960-8972.

47. Y. Gao, J. Liu, J. Shen, L. Zhang, Z. Guo and D. Cao, Physical Chemistry Chemical Physics, 2014, 16, $16039-16048$.

48. C.-C. Huang, R. G. Winkler, G. Sutmann and G. Gompper, Macromolecules, 2010, 43, 1010710116.

49. Y. Gao, D. Cao, J. Liu, J. Shen, Y. Wu and L. Zhang, Physical Chemistry Chemical Physics, 2015, 17, 22959-22968.

50. D. Wan, Z. Zhang, Y. Wang, H. Xing, Z. Jiang and T. Tang, Soft Matter, 2011, 7, 5290-5299.

51. C. Baig, B. J. Edwards, D. J. Keffer, H. D. Cochran and V. A. Harmandaris, The Journal of Chemical Physics, 2006, 124, 084902.

52. A. Jabbarzadeh, J. D. Atkinson and R. I. Tanner, Macromolecules, 2003, 36, 5020-5031.

53. W. Chen, J. Chen, L. Liu, X. Xu and L. An, Macromolecules, 2013, 46, 7542-7549. 


\section{Table of Content Graphics}

Haoxiang $\mathrm{Li}^{1,2,3}$, Haoyu Wu ${ }^{1,2,3}$, Wenfeng Zhang ${ }^{1,2,3}$, Xiuying Zhao ${ }^{1,2,3}$, Yangyang Gao ${ }^{1,2,3^{*}}$, Liqun Zhang $^{1,2,3}$

Rheological behavior of polymer nanocomposites filled with spherical nanoparticles: Insights from molecular dynamics simulation

* Corresponding author: gaoyy@ mail.buct.edu.cn 


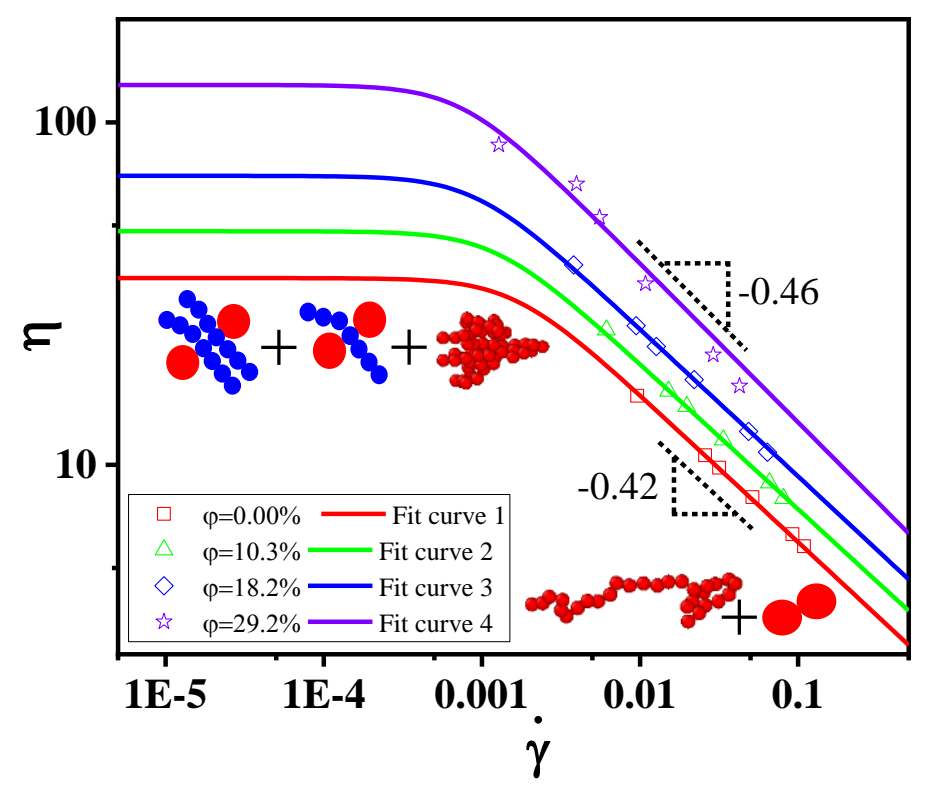

